\title{
Engineering of bacterial strains and their products for cancer therapy
}

\author{
Nuno Bernardes ${ }^{1}$, Ananda M Chakrabarty ${ }^{2}$ and Arsenio M Fialho ${ }^{1}$
}

${ }^{1}$ Institute for Biotechnology and Bioengineering (IBB), Center for Biological and Chemical Engineering, Instituto Superior Técnico, 1049-001 Lisbon, Portugal

${ }^{2}$ Department of Microbiology \& Immunology, University of Illinois College of Medicine, Chicago, IL 60612, USA

Author for correspondence: Arsenio M. Fialho - afialho@ist.utl.pt

Key words: cancer, bacteria, immunotherapy, azurin, metagenomics 


\section{Abstract}

The use of live bacteria in cancer therapies offers exciting possibilities. Nowadays, an increasing number of genetically engineered bacteria are emerging in the field, with applications both in therapy and diagnosis. In parallel, purified bacterial products are also gaining relevance as new classes of bioactive products to treat and prevent cancer growth and metastasis. In the first part of the article, we review the latest findings regarding the use of live bacteria and products as anti-cancer agents, paying special attention to immunotoxins, proteins and peptides. In particular, we focus on the recent results of using azurin or its derived peptide as anticancer therapeutic agents. In the second part, we discuss the challenges of using metagenomic techniques as a distinctive approach for discovering new anticancer agents from bacterial origin. 


\section{Introduction}

Cancer therapy is often challenged with secondary effects caused by standard therapies and frequently faces tumor cell resistance and the inability to eliminate micrometastases (Morrissey et al. 2010). Therefore, new therapies are urgently needed. Recently, there has been renewed interest in the development of new therapeutic anticancer modalities based on the use of live bacteria and their purified products. In late-nineteenth-century, bacteria were discovered as anticancer agents by the surgeon William B. Coley, who first observed regression in tumors after some of his patients were infected with bacteria. The so-called Coley's toxins were used against different types of cancer, but despite anti-tumor activity, a few patients developed systemic infections and eventually died (Chakrabarty 2003). Nowadays, the problems with systemic infections after bacterial delivery are being overcome either by using engineered attenuated bacteria with low infection capabilities or bacterial products which are capable per se of targeting and specifically killing tumor cells (Fig. 1). Bacteria as gene delivery vectors (Forbes 2010) or the use of bacterial products engineered to more tailored approaches in cancer treatment are currently being investigated for proof-ofconcept and are already in the early phases of clinical trials to enter in clinical practice. Specific targeting of cancer cells would then allow the use of more cytotoxic products without undesired toxicity to normal tissues.

The purpose here is to update on the most recent developments since publication of our previous review (Bernardes et al. 2010) regarding the use of live bacteria and their products as anti-cancer agents. Furthermore, we outline the 
recent achievements and future directions in using metagenomics techniques as innovative routes for anticancer drug discovery from bacterial origin. We believe that the bacteria living in inhospitable environments on earth are a hidden source of novel bioactive anticancer drugs that need to be explored.

\section{Attenuated bacterial strains: specific tumor targeting and gene therapy of cancer}

Initially, the capacity of bacteria to target cancer cells was attributed to anaerobic or facultative species due to the hypoxic nature of the tumor microenvironment. However, recent studies conducted on mice indicate that the oxygen requirement of bacteria doesn't determine if bacteria will colonize the interior or the exterior of solid tumors (Yu et al. 2008). Additionally, Yu et al (2008) conducted a study where both Gram-negative and Gram-positive bacteria were capable of colonizing tumor tissues after being given intravenously to mice. This work also established the optimal dosing of live bacteria $\left(10^{4}-10^{5}\right.$ cells) and defined limiting factors for treatment, namely the stage of tumor progression and a suitable nutritional environment for the bacteria. In general, the efficacy of the anticancer treatment is independent of tumor type, thereby suggesting that bacterial entry into the tumors is not mediated by a specific tumor-cell receptor (Yu et al. 2008). Regardless of these findings, in the last decade members of the genera Clostriudium, Salmonella and Bifidobacterium have been the most studied vectors for gene and cell therapy targeting tumors (Fialho and Chakrabarty 2010). Animal models have contributed greatly to the progress of such studies, revealing 
promising results and providing insights into anticancer mechanisms. Several clinical trial studies are currently undergoing and the next step will be the translation of these approaches into effective therapies for humans.

Oncolytic activity

Oncolytic strains such as Clostriudium and Salmonella show the ability to kill cancer cells due to their own replication in tumor cores (Baban et al. 2010). The strictly anaerobic Clostridium novyi ATCC 19402 was engineered by deletion of a gene coding for the $\alpha$-toxin (so-called C. novyi-NT) (Dang et al. 2001). Following intravenous administration in tumor-bearing mice, this attenuated strain preferentially replicated within specific tumor microenvironment, ultimately causing tumor cell death (Dang et al. 2001). Injection of C. novyi-NT spores are being investigated in animal tumor models in combination with conventional chemotherapeutics - COBALT (combination bacteriolytic therapy). More recently, lipomase, an enzyme produced by $C$. novyi-NT with membrane-disrupting capacities was identified and a new approach was adopted by releasing liposomeencapsulated lipomase within tumors (Cheong et al. 2007). Mice bearing tumors treated with C. novyi-NT plus a single dose of liposomal doxorubicin resulted in significant inhibition of tumor growth.

Several genetic alterations have also been made in Salmonella with the aim of creating an attenuated strain that could be safely administered into humans and take advantage of its natural ability to direct itself to tumors. Salmonella typhimurium VNP2009 is an attenuated strain devoid of two genes, namely, $m s b B$ and purl. These genetic alterations prevent replication in healthy organs such as 
the liver or spleen and introduced the need to external sources of purines, which can be obtained in the tumor external media. The clinical safety of this strain has already been shown in clinical trials (Nemunaitis et al. 2003).

Delivery of cytotoxic genes

The use of live attenuated bacteria as vectors to deliver cytotoxic genes is another attractive approach. Selective localization within tumors makes these vectors attractive to deliver anticancer agents, therapeutic peptides/proteins and prodrugs that can be activated by corresponding converting enzymes. Clostridium acetobotylicum and Clostridium sporogenes expressing cytosine deaminase (CD) and nitroreductase (NTR) significantly delayed tumor progression (Burke et al. 2006; Liu S-C 2002; Theys et al. 2001). CD promotes tumor site-specific conversion of 5-fluorocytosine (5-FC) prodrug into its active form (5-fluorouracil, 5FU) whereas NTR catalyzes the reduction of the prodrug metrodinazole (Mtz) thereby producing a toxic compound. A genetically engineered Salmonella typhimurium VNP2009, named TAPET-CD, was used to express CD with clinical safety demonstrated in Phase I trials (Morrissey et al. 2010). Expression of various others therapeutic proteins, such as TNF- $\alpha$, platelet factor 4 fragments and TRAIL was achieved in the VNP2009 strain, leading to the regression of xenografted tumors (Burke et al. 2006; Liu S-C 2002). Expression of CD by Bifidobacterium longum in combination with endostatin expression or low dose adryomicin improved tumor regression in S180 osteosarcoma bearing mice (Xu et al. 2007). 


\section{Up-regulation of the immune system}

The use of live bacteria is emerging as an immunotherapeutic strategy to treat certain types of cancer (Bolhassani and Zahedifard 2012). Often, human immune system recognizes cancers as self-antigens or the cancers are weakly immunogenic, so the presence of bacteria in the tumor environment is likely to increase the immune response necessary to overcome these barriers. Furthermore, to enhance the immune response, live bacteria can also be engineered to deliver pro-immune proteins, such as granulocyte colony-stimulating factor (G-CSF) (Uhua et al. 2001).

Immunotherapy with Mycobacterium bovis Bacillus Calmete-Guerin (BCG) is used in clinical practice to treat/prevent the recurrence of superficial bladder cancer (Kresowik and Griffith 2010). The choice of a BCG based therapy depends upon the location and grade of the tumor and follows the recommendations described by several organizations, such as the ESMO Clinical Practice Guidelines (Bellmunt et al. 2010). Moreover recently it was shown that BCG can have a radiosensitizing effect on colon cancer cells, thereby revealing the use of this bacterium as a new immunotherapy option for this type of cancer (Yuk et al. 2010).

Other attenuated bacteria have been used to promote the up-regulation of the immune system such as Clostridium, Salmonella, Bifidobacteria and Listeria (Fu et al. 2010; Hoffman 2010; Mellaert et al. 2010; Rothman et al. 2010). C. novyi-NT spores potentiate an inflammatory response in the tumor microenvironment resulting in enhanced cytokine production such as IL-6, MIP-2, G-CSF and TIMP-1 (Xu et al. 2009). These agents help recruit to the tumor site multiple inflammatory 
cell types, which in turn produce a dual effect by controlling the bacterial infection and targeting cancer cells leading to cancer regression (Patyar et al. 2010).This approach led to the design of phase I clinical trials, either with the spores alone or in combination with antimicrotubule agents (Xu et al. 2009).

S. typhimurium was engineered to express several human proteins with immunotherapeutic properties, resulting in tumor reduction due to growth of the bacteria in the tumor. Reports of expression of IL-8, CCL21, LIGHT or the Fas ligand have recently been published (Hoffman 2010). The facultative intracellular bacterium Listeria monocytogenes is being studied for the development of cancer vaccines. Recombinant strains expressing a nucleoprotein from influenza strain A/PR8/34 (Lm-NP), the truncated listeriolysin (LLO) or the fusion of this protein with HPV16 E7 protein have all shown good preclinical results and have been used to perform several clinical trials (Patyar et al. 2010).

\section{Gene therapy using bacteria}

Bacteria can be used as a vector to deliver genetic material into mammalian cells, in a process denominated as bactofection. L. monocytogenes, E.coli and Salmonella spp. have been used to mediate gene transfer as DNA vaccines-based vectors. Endostatin gene therapy delivered by Salmonella choleraesuis in murine tumor models has been proved effective (Xu et al. 2007). S. choleraesuis carrying thrombospondin-1 (TSP-1) gene for treating primary melanoma and experimental pulmonary metastasis in the syngeneic murine B16F10 melanoma model significantly inhibited tumor growth and enhanced survival of the mice, displaying 
decreased intratumoral microvessel density (Lee et al. 2005). An attenuated $S$. typhimurium strain harboring a recombinant plasmid carrying both TRAIL and Smac (also known as DIABLO) genes under the control of the hTERT promoter, is a promising antitumor strategy. This engineered strain was orally administered into mice bearing tumors, and its antitumoral effect was evaluated with tumor growth inhibition by $70-90 \%$ and prolonged survival of mice (Fu $\mathrm{W}$ et al. 2008). In vivo Salmonella-mediated exogenous gene expression persisted for at least 14 days in tumors. In vitro results showed that Smac could enhance TRAIL-induced apoptosis in tumor cells and the hTERT promoter controlled specific gene expression in tumor cells, but not in normal cells. Another strategy exploits the use of Salmonella as a vector system to deliver plasmids encoding cytokines, such as IL-2 and GMCSF. This strategy has proved effective in inhibiting tumor growth with a reduced overall systemic toxicity (Uhua et al. 2001).

An ampicillin sensitive strain of $L$. monocytogenes has been used to deliver DNA to cancer cells. Bacteria can be lysed through systemic administration of ampicillin thereby facilitating the release of the therapeutic DNA in the tumor microenvironment (Tangney et al. 2010).

Engineered E. coli cells expressing the invasin gene (inv A) from Yersinia pseudotuberculosis selectively invade nonphagocytic cells in which $\beta 1$-integrin is expressed. Co-expression of listeriolysin (LLO) gene mediates the release of the content of the bacteria into the cytosol of the invaded cell. Bacteria failed to invade normal epithelial cells of the gut due to the inaccessible localization of $\beta 1$-integrin. Invasive $E$. coli cells co-expressing the model antigen ovalbumin and LLO from $L$. monocytogenes were orally administered in mice which prompted systemic 
protection against B16 tumor cells expressing ovalbumin (Critchley-Thorne et al. 2006).

\section{Bacteria for Bioimaging}

One of the most difficult tasks in cancer treatment is the identification of small disperse metastasis or small tumors. The ability of bacteria to colonize these tumors is clinically important because engineered bacteria can be generated enabling the detection of these cancer cells (Cronin et al. 2012; Forbes 2010). Imaging can be achieved by bioluminescence, fluorescence, magnetic resonance or positron emission. Light based mechanisms, either bioluminescence or fluorescence can be obtained with plasmids containing the luxCDABE gene cassete derived from Photobacterium leiognathi or florescence-encoding genes like GFP, respectively, however these may have clinical disadvantages due to the poor penetration of visible light through tissues (Forbes 2010). Magnetospirillum magneticum produces magnetic particles which after tumor colonization accumulate within cancer cells, allowing positive magnetic resonance imaging contrast (Benoit et al. 2009). Different mouse tumor models have been tested using either endogenous or exogenous tyrosine kinase activities from bacteria: Salmonella expressing the herpes simplex thymidine kinase (HSV1-TK) gene phosphorylates the marker 2'-fluoro-1- $\beta$-D-arabino-furanosyl-5-iodouracil (FIAU), enabling the detection of bacteria inside tumors (Soghomonyan et al. 2005); also, endogenous kinases from E.coli Nissle 1917 phosphorylates $\left[{ }^{18} \mathrm{~F}\right] 2$ '-fluoro-2'deoxy-1- $\beta$-D-arabino-furanosyl-5-ethyl-uracil $\left(\left[{ }^{18} \mathrm{~F}\right]-\mathrm{FEAU}\right)$ and can be used to 
detect tumors (Brader et al. 2008).

\section{Bacterial products for cancer treatment}

Not only live attenuated bacterial strains have been proposed as anticancer agents but also products derived from them such as enzymes, secondary metabolites, proteins or derived peptides and toxins (Fig. 1) (Bernardes et al. 2010).

Bacterial toxins are amongst the most cytotoxic products in nature (Pastan et al. 2007). New strategies, by genetic and protein engineering, offer the possibility to fuse these toxins with monoclonal antibodies (termed immunotoxins), creating new powerful chimeric proteins that specifically target cancer cells (Weldon and Pastan 2011). Bacterial toxins possess defined domains which allow their use in cancer therapy: a cell recognition (binding) domain to allow for higher concentrations of the toxin around target cells, a translocation domain for cell internalization and a death domain to exert potent cytotoxic effects. For the therapeutic immunotoxins, the natural cell recognition domain is replaced by a new ligand towards a specific receptor directing the toxin to a particular subset of cancer cells (Pastan et al. 2007; Weldon and Pastan 2011). Exotoxin A from Pseudomonas aeruginosa (PE) and diphtheria toxin (DT) from Corynebacterium diphtheria are the most commonly used toxins for cancer therapy (LorberboumGalski 2011). These toxins kill human cells by preventing protein synthesis after inactivation of the elongation factor EF-2 through ADP ribosylation. First generations of these drugs were made by chemical coupling, but nowadays 
immunotoxins are made by DNA recombination to fuse at the genetic level the killer domain to a cytokine or growth factor for cell recognition and binding (Pastan et al. 2007). The use of these products due to their cytotoxic nature has been strongly accompanied by several modifications which adapted them to a safer use in humans (Choudhary et al. 2011).

$\mathrm{PE}$, the $P$. aeruginosa exotoxin $\mathrm{A}(66 \mathrm{kDa}, 638$ amino acids) contains four functional domains: the receptor binding domain (la; aa 1-252), the translocation domain (II; aa 253-364), the domain Ib (aa 365-404) with an unknown function and the cytotoxic domain (aa 405-613) (Wolf and Elsässer-Beile 2010). Mostly, PEbased immunotoxins use a truncated version of $\mathrm{PE}$ after removing its $\mathrm{N}$-terminal receptor binding domain. Additionally, another mutant form of PE, lacking the receptor binding domain together with 16 amino acids from domain $\mathrm{lb}$, is also successfully used (named PE 38). Several clinical trials have been performed based on PE fused to different ligands. As an example, BL22 against CD22 and LMB-2 against CD25 are two PE38-based toxins with good results in patients with hematologic tumors (Wolf and Elsässer-Beile 2010).

Ontak $^{\mathrm{TM}}\left(\mathrm{DAB}_{389} \mathrm{lL2}\right)$ is the first immunotoxin approved by the FDA for the treatment of T-cell lymphoma. It is based in a truncated form of DT spanning amino acids 1-389 and targets IL2 receptor (Choudhary et al. 2011). IL2 receptor has been shown to be expressed in other cancers such as melanoma, renal cell carcinoma, head and neck carcinomas, esophageal and lung cancers (Huang et al. 2002; Tartour et al. 2001; Wang et al. 2000). Other ligands have also been used with this toxin and different combinatorial therapies as well. A more extensive list of clinical trials in solid and hematological tumors using these toxins has been 
compiled (Choudhary et al. 2011).

During the last years, a number of bacterial proteins and peptides have been described to exert an anticancer activity at pre-clinical level towards diverse types of cancer cells. These proteins or peptides are found in unrelated bacteria with anticancer activity. In this section we summarize current information about known bacterial proteins/peptides that could potentially be used to develop new treatments against cancer. A special emphasis is given to azurin protein and its derived peptide p28.

SSL10 is a superantigen like protein from Staphylococcus aureus and it inhibits the $\mathrm{CXCl12-induced} \mathrm{migration} \mathrm{of} \mathrm{leukemic} \mathrm{Jurkat} \mathrm{cell} \mathrm{line} \mathrm{and} \mathrm{carcinoma}$ cell line Hela. CXCL12 is a ligand of CXCR4 and binding of SSL10 to the receptor prevented the binding of the natural ligand and migration. Also SSL5 has a role in preventing adhesion of leukemic cells to endothelial cells and platelets. SSL5, another superatingen like protein from S. aureus binds to P-selectin glycoprotein-1 that is expressed in HL-60 cells and impedes the binding to endothelial cells (HUVEC). Interactions between tumor cells and endothelial cells are important for tumor progression since it mediates processes such as angiogenesis and metastasis formation (Tumor et al. 2009; Walenkamp et al. 2010).

The Actin assembly-inducing protein (ActA) plays an important role in pathogenesis of Listeria monocytogenes. Once the bacteria are internalized into host cells, ActA induces a rapid polymerization of actin filaments by interacting with the Arp2/3 complex (Wood et al. 2010). After this, the protein is rapidly degraded by the ubiquitin-proteasome machinery. Besides its natural role, ActA promotes 
tumor cell killing by immune mechanisms. ActA has been fused to a tumor antigen to drive adjuvancy in tumor immunotherapy. Moreover, it is now known that the protein alone, with no antigen fused, has the same ability, providing a $C D 8^{+}$celldependent anti-tumor immune response. It has been proposed that ActA may acts as a PAMP (pathogen associated molecular pattern), like other bacterial adjuvants, such as LLO, widely used in immunotherapy to facilitate CD8-mediated immune responses (Wood et al. 2010).

Romidepsin (FK228) is a naturally occurring bicyclic dipeptide isolated from Chromobacterium violaceum which acts as histone deacetylase inhibitor (HDI). Histone deacetylases are implicated in leukemia development and progression, and therefore are important therapeutic targets in this malignancy (Vinodhkumar et al. 2008). Spiruchostatin B (SP-B) is a structurally related peptide isolated from a culture broth of Pseudomonas $s p$ which displays the same activity towards cancer cells (Kanno et al. 2012). Addition of SP-B to NALM-6 human B cell leukemia cells at concentrations $\geq 6 \mathrm{nM}$ led to an increase of both mRNA and protein expression of p2 $1^{\text {wat/cip1 }}$ which has a particular high expression in this cell line. This protein is a known regulator of cell cycle and modulator of apoptosis and is used as a marker of histone deacetylases inhibition. The increase in this protein was parallel to a cell cycle arrest of leukemia cells with an increased population at $G_{0} / G_{1}$ phase and increased apoptosis, proving that this family, particularly in these cells with high expression levels of $\mathrm{p} 21^{\text {wat/cip } 1}$ are suitable targets for SP-B.

Pep27anal2 is an analogue of Pep27, a peptide from Streptococcus pneumoniae where it initiates a program of cell death by signal transduction mechanisms (Lee et al. 2005). This and other cationic peptides are studied for their 
possible effects in cell death induction due to their ability to disrupt cell membranes. Despite the fact that Pep27 did not show anticancer activity at concentrations upto $70 \mu \mathrm{M}$, peptide engineering increased this effect by amino acid substitutions. Four amino acids $\left({ }^{2} \mathrm{R},{ }^{4} \mathrm{E},{ }^{11} \mathrm{~S}\right.$ and $\left.{ }^{13} \mathrm{Q}\right)$ were substituted by trypthophans increasing the hydrophobicity and therefore the anticancer activity at concentrations of about $30 \mu \mathrm{M}$ (Lee et al. 2005). The mechanism by which Pep27anal2 penetrates the membranes is not fully understood but it is known that once inside it causes apoptosis, displaying typical markers such as exposition of phosphatidyl serine in the outer membrane, chromatin condensation but not cytochrome c release from mitochondria, suggesting that a mitochondriaindependent mechanism is initiated by Pep27anal2. The substitutions performed in Pep27anal2 revealed that this peptide acquires a more stable $\alpha$-helical conformation which is proposed to be related to the higher anticancer activity by promoting membrane penetration.

Azurin, a small copper protein produced by $P$. aeruginosa, can act as an anticancer agent. It combines antiangiogenic and tumor cell cytotoxic effects (Punj et al. 2004; Yamada et al. 2004; Yamada et al. 2002; Zaborina et al. 2000). Laz is an azurin-like protein produced by gonococci/meningococi. Unlike other azurins, it is surface exposed and harbors an additional $\mathrm{N}$-terminal epitope of 39 amino acids (Fialho et al. 2012b). Several US patents have been issued to cover the use of azurin and Laz in cancer therapies [for a recent update see (Fialho et al. 2012a)], and azurin has shown significant activity, as well as enhancement of the activity of other drugs, in oral squamous carcinoma cells (Choi JH et al, 2011. Yonsei Med. J. 52: 773-778). 
It has been demonstrated that azurin can directly interact and stabilize the tumor suppressor p53. Following treatment with azurin, the p53 wild-type MCF-7 breast cancer cells showed an increase in p53 levels in both nuclear and cytoplasmatic fractions (Punj et al. 2003; Yamada et al. 2005; Yamada et al. 2004). The presence of azurin is likely to increase the mRNA levels of pro-apoptotic molecules via p53, such as the levels of $B A X$, thereby leading to an unbalance of BCL2-BAX levels leading to increased cell death or growth arrest (Punj et al. 2003).

Yamada et al (2005) identified the azurin domain responsible for its specific entry in cancer cells. It spans residues 50-77 (termed p28) and adopts an amphipathic alpha-helical conformation (Yamada et al. 2005). In contrast with most of the cell-penetrating peptides, p28 has an overall net negative charge (Taylor et al. 2009). The mechanism mediating cell entry of azurin and its derived peptide has been studied. It is known that it is not dependent on membrane bound glycosaminoglycans nor on clathrins. However, it is possible that N-glycosylated proteins may have a role at least in the initial steps of recognition and the depletion of cholesterol from the membrane significantly inhibited the penetration of p28 ( $60 \%)$, suggesting involvement of the caveolae-mediated endocytic route (Taylor et al. 2009). Cell penetration is not accompanied by membrane disruption, which could cause cell death per se, and is energy-dependent since penetration occurs much faster at $37^{\circ} \mathrm{C}$ than at $4^{\circ} \mathrm{C}$. Preclinical evaluation of pharmacokinetics, metabolism and toxicity of azurin-p28 was evaluated (Jia et al. 2011), establishing it as non-immunogenic and non-toxic in mice and non-human primates.

The interactions between azurin and p53 have recently been analyzed, 
namely by bioinformatic and protein-protein interactions, using Atomic Force Microscopy (AFM) (De Grandis et al. 2007; Taranta et al. 2008; Taranta et al. 2009). Azurin harbors the possibility to bind to the various domains of $p 53$ and multiple configurations are possible to occur. Moreover, azurin bearing mutations in Met-44 and Met-64 residues were analyzed by Molecular Dynamics simulations. A large loop of p53 DNA Binding Domain $\left(L_{1}\right)$ and the mentioned hydrophobic amino acids of azurin are indeed in the interface and the loop underwent structural adaptations which led to a better packing and constructive van der Waals contacts. Simulations performed with azurin M44KM66E weakened the above observations in the models obtained, in accordance to the biological evidences, previously stating that these mutants were less cytotoxic than wild type azurin (Yamada et al. 2004). Also, the peptide p28 demonstrates the ability to bind itself to $p 53$ both by Molecular Dynamics simulations (Santini et al. 2011) and atomic force spectroscopy (Bizarri et al. 2011).

p28 peptide has undergone phase I clinical trial supported by CDG Therapeutics Inc. (http://www.cdgti.com/; IND 77,754) (Fig. 2). Fifteen stage IV cancer patients with metastatic refractory tumors resistant to conventional drugs and with no more than 6 months of life expectancy were included in this trial covering different cancer types (7 melanoma, 4 colon, 2 sarcoma, 1 pancreatic and 1 prostate). In none of these patients, any significant toxicity was observed and 2 patients demonstrated partial regression while 2 others demonstrated complete regression of their tumors (Yamada T et al. 2011), demonstrating a unique mode of action of p28.

Interestingly, the bacterial protein azurin demonstrates the ability to bind 
multiple targets in mammalian cells, both extra- and intracellular. Besides its interaction with p53, azurin also targets a cell proliferation pathway mediated by the EphB2 tyrosine kinase. It was demonstrated that azurin exhibits a competitive binding towards this receptor being able to prevent the tumor progression caused by the binding of the natural ligand ephrinB2. EphB2 is overexpressed in several types of cancer and the drugs available in the market targeting these receptors act through the ATP binding pockets of the kinases. Such a mode of binding is often associated with lack of specificity, inhibiting other physiologically important kinases not related to cancer and creating toxicity problems to the patients. Azurin, on the other hand, displays structural similarities to ephrinB2, and thereby binds EphB2. The region of azurin responsible for the interaction is a G-H loop, spanning from amino acids $88-113$ and this is similar to the loop in the ephrinB2 ligand that mediates the recognition to the receptor (Chaudhari et al. 2007). In addition, preferential entry of azurin and its inhibition of the phosphorylation of VEGFR-2, FAK and Akt allows inhibition of angiogenesis in cancer cells, thus inhibiting cancer cell growth (Mehta et al, 2011).

The ability of azurin to bind to Ephrin receptors has been used to engineer a peptide conjugated to nicotinamide to increase sensitivity to radiotherapy (Fig. 2). A small library based on the fragment of azurin comprising amino acids 88-128 was created and screened by Surface Plasmon Resonance to obtain the best binding affinities to Eph receptors EphA2, EphB2 and EphB4. Modifications such as the introduction of additional charged residues or increased hydrophobicity were tested to improve affinity. The element of the library with the best properties was further modified to enhance solubility and stability at physiological conditions and 
conjugated to nicotinamide, AzV36-Nic, or a linear form, AzV36-NicL which displayed the best binding affinities in the nanomolar range (Micewicz et al 2011). The sensitizing activity of this derived peptide was assessed in two in vivo models, an artificial metastasis model and solid tumor engraftment model. In both cases the presence of the peptide increased the efficacy of radiotherapy with the results achieving a $\sim 13$ fold increase in the efficacy of the treatment (Micewicz et al 2011).

Bacterial therapy may have a major advantage to allow intratumoral production of cytotoxic drugs, which in turn would be more toxic to cancer tissues and less toxic to normal cells (Forbes 2010). Very recently, a combination of the azurin therapeutic effects and bacterial anticancer activity was demonstrated through the administration of E.coli Nissle 1917 (EcN) expressing azurin (Fig. 2). Safe doses of this bacterium were given to mice with no visible toxic effects and preferential accumulation within the necrotic areas of the tumor. When injected to mice bearing B16 melanoma or $4 \mathrm{~T} 1$ breast tumor models, $2 \times 10^{7} \mathrm{CFU}$ per mouse caused a marked delay in tumor progression and prolonged survival of the mice by about $50 \%$. Moreover, $4 \mathrm{~T} 1$ is a highly metastatic tumor, particularly for the lungs. With EcN therapy the number of metastatic nodes was clearly reduced by about $40 \%$ after 30 days of the treatment when compared to control groups of either PBS or EcN not expressing azurin (Zhang et al. 2012).

The perspectives of using azurin in combination with recent technologies developed in the nanomedicine field have recently been analyzed (Keyhanian et al. 2010). In fact, not only azurin, but other proteins or peptides originated from bacteria such as those described above, can be tested in new ways including gold nanoparticles, magnetic nanoparticles, dendrimers, folic acid or carbon nanotubes 
in an attempt to improve their bioavailability or efficacy (Keyhanian et al. 2010). Moreover, such products can be packaged in bacterial minicells, representing nonliving structures derived from a mutant strain of Salmonella enterica serovar Typhimurium. These nano-cellular carriers have been successfully used to deliver drugs to target cancer cells (MacDiarmid and Brahmbhatt 2011).

\section{Exploring the microbial world through metagenomics: new ways of detecting bioactive molecules for cancer therapy}

The medicine of the $21^{\text {st }}$ century urgently needs new tools and strategies to help in the discovery of novel drugs for cancer treatment. Many biopharmaceutical companies and university laboratories have invested in sophisticated technologies that integrate scientific knowledge on genomics, proteomics, molecular modeling, combinatorial chemistry, automated high-throughput screenings and computational docking. Among those, metagenomics is emerging as a powerful technology to discover novel biologically active molecules, such as antibiotics, anticancer agents, immunosuppressants, as well as a large variety of enzymes and other biotechnologically valuable products.

Metagenomics has been successfully used to study complex microbial communities or to assess microbial colonization in environments that cannot be mimicked in conventional laboratory conditions. In the past few years, the scientific advancements enable its use in the field of drug discovery. In fact, nowadays, metagenomics offers the possibility to explore the vast unknown microbial world as a source of potential new anticancer drugs (Fig. 3). First, this approach involves 
the direct extraction of genetic material from inhospitable environments, such as soil samples taken from caves or volcanic regions or deep-sea sediment samples. It is believed that the resident microbial communities in those extreme terrestrial and marine environments represent a promising source of novel leads for the development of anticancer agents. Among various established protocols, the genomic DNA extracted is then ligated into a phagemid vector aiming to create phage display libraries. Using such approach, the DNA from thousands of environmental microbes could be expressed and displayed on the phage surface. Then, through an affinity selection technique, the phage libraries are subjected to three or four rounds of panning against selected targets for cancer therapy. Of particular interest amongst these are P-cadherin, VEGF, EGFR, CD20 receptor and ephrin receptors. In a secondary step, the clones identified can be analyzed by DNA sequencing followed by their expression and purification in a suitable bacterial host. Finally, in vitro and in vivo experiments are used to validate the new anticancer lead compounds (Fig. 3). Afterwards, once a bioactive compound is discovered, further optimization in terms of binding and specificity for a particular cancer target is followed. For this purpose, combinatorial phage display libraries can be used to engineer novel proteins that bind strongly to a cancer specific target. This approach can be reached by randomized substitution of amino acids of selected residues within pre-existing binding sites of the lead compounds.

Few studies have as yet been conducted using metagenomes as sources of novel therapeutic molecules (Rath et al. 2011; Zago et al. 2008). However, metagenomics is increasingly being viewed as a powerful technique for discovering new bioactive therapeutic molecules. Recently, a particular emphasis has been 
given to the exploitation of the human microbiome, particularly the intestinal microbiome (Schloissnig et al. 2013). We anticipate that such metagenomic approaches, when fully researched, will represent a valuable strategy for the discovery of novel and promising bioactive molecules with anticancer activity.

\section{Final remarks and future perspectives}

Cancer is a leading cause of death in the world (about 7.6 million people died of cancer in 2008) (Globocan 2008, IARC, 2010). Based on World Health Organization (WHO) projections, in 2030, the number of people expected to die of cancer will be around 11.4 million. Despite the enormous amount of resources devoted to the area of drug development, cancer treatment remains one of the biggest challenges in public health. Many drugs have been developed to treat cancer. Most of them come from high-throughput chemical library screening (HTS) and are designed to target specific oncogene products involved in cancer progression. However, after exposing tumor cells to those anticancer drugs, both toxicity and the development of resistance are major reasons for failure in cancer therapy (Avner et al. 2012). Therefore, new paradigms for cancer drug development are urgently required. It's interesting and somewhat surprising that bacteria may hold the key for finding new therapeutic approaches against cancer. In this article, we have presented an overview of this innovative field of research, involving live engineered microorganisms or their products as new promising anticancer agents. 


\section{Conflict of interest}

The authors declare no conflict of interest.

\section{Acknowledgements}

N.B. acknowledges a PhD grant (SFRH/BD/48763/2008) from Fundação para a Ciência e a Tecnologia (FCT). Research in the A. M. Fialho lab was supported by FCT (Grant PTDC/EBB/BIO/100326/2008).

\section{References}

Avner BS, Fialho AM, Chakrabarty AM (2012) Overcoming drug resistance in multi-drug resistant cancers and microorganisms: a conceptual framework. Bioengineered 3(5):262-270. doi:10.4161/bioe.21130

Baban CK, Cronin M, O'Hanlon D, O'Sullivan GC, Tangney M (2010) Bacteria as vectors for gene therapy of cancer. Bioengineered 1:385-94. doi: 10.4161/bbug.1.6.13146

Bellmunt J, Orsola A, Maldonado X, Kataja V (2010) Bladder cancer: ESMO practice guidelines for diagnosis, treatment and follow-up. Ann Oncol 21(suppl 5):v134-v136. doi: 10.1093/annonc/mdq175

Benoit MR, Mayer D, Barak Y, Chen IY, Hu W, Cheng Z, Wang SX, Spielman DM, Gambhir SS, Matin A (2009) Visualizing implanted tumors in mice with magnetic resonance imaging using magnetotactic bacteria visualizing implanted tumors in mice with magnetic resonance imaging using magnetotactic bacteria. Clin Cancer Res 15:5170-5177. doi: 10.1158/1078-0432.CCR-08-3206

Bernardes N, Seruca R, Chakrabarty AM, Fialho AM (2010) Microbial-based therapy of cancer: current progress and future prospects. Bioengineered 1:178-90. doi: 
10.4161/bbug.1.3.10903

Bizarri AR, Santini S, Coppari E, Bucciantini M, D Agostino S, Yamada T, Beattie CW, Cannistraro S (2011) Interaction of an anticancer peptide fragment of azurin with p53 ad its isolated domains studied by atomic force spectroscopy. Int J Nanomed 6: 30113019. doi: 10.2147/IJN.S26155

Bolhassani A, Zahedifard $F$ (2012) Therapeutic live vaccines as a potential anticancer strategy. Int J Cancer 131(8):1733-1743. doi: 10.1002/ijc.27640

Brader P, Stritzker J, Riedl CC, Zanzonico P, Cai S, Burnazi EM, Ghani ER, Hricak H, Szalay A a, Fong Y, Blasberg R (2008) Escherichia coli Nissle 1917 facilitates tumor detection by positron emission tomography and optical imaging. Clin Cancer Res 14:2295-302. doi: 10.1158/1078-0432.CCR-07-4254

Burke PJ, Theys J, Pennington O, Dubois L, Anlezark G, Vaughan T, Mengesha A, Landuyt W, Anne J (2006) Repeated cycles of Clostridium-directed enzyme prodrug therapy result in sustained antitumour effects in vivo. Brit J Cancer 95(9):1212-1219. doi: 10.1038/sj.bjc.6603367

Chakrabarty AM (2003) Microorganisms and cancer: quest for a therapy. J Bacteriol 185:2683-2686. doi: 10.1128/JB.185.9.2683

Chaudhari A, Mahfouz M, Fialho AM, Yamada T, Granja AT, Zhu Y, Hashimoto W, Schlarb-Ridley B, Cho W, Das Gupta TK, Chakrabarty AM (2007) Cupredoxin-cancer interrelationship: azurin binding with EphB2, interference in EphB2 tyrosine phosphorylation, and inhibition of cancer growth. Biochemistry 46:1799-810. doi: 10.1021/bi061661x

Cheong I, Huang X, Thornton K, Diaz LA, Zhou S (2007) Targeting cancer with bugs and liposomes: ready, aim, fire. Cancer Res 67:9605-8. doi: 10.1158/0008-5472.CAN-071565

Choudhary S, Mathew M, Verma RS (2011) Therapeutic potential of anticancer immunotoxins. Drug Discov Today 16:495-503. doi: 10.1016/j.drudis.2011.04.003

Critchley-thorne RJ, Stagg AJ, Vassaux G (2006) Recombinant Escherichia coli expressing invasin targets the Peyer's patches: the basis for a bacterial formulation for oral vaccination. Mol Ther 14:183-191. doi: 10.1016/j.ymthe.2006.01.011

Cronin M, Akin AR, Collins S a, Meganck J, Kim J-B, Baban CK, Joyce S a, Van Dam GM, Zhang N, Van Sinderen D, O'Sullivan GC, Kasahara N, Gahan CG, Francis KP, Tangney M (2012) High resolution in vivo bioluminescent imaging for the study of bacterial tumour targeting. PloS One 7:e30940. doi: 10.1371/journal.pone.0030940

Dang LH, Bettegowda C, Huso DL, Kinzler KW, Vogelstein B (2001) Combination bacteriolytic therapy for the treatment of experimental tumors. PNAS 98:1515515160. 
Fialho AM, Bernardes N, Chakrabarty AM (2012a) Recent patents on live bacteria and their products as potential anticancer agents. Recent Patents on Anti-Cancer Drug Discovery 7:31-55. doi: 10.2174/157489212798357949

Fialho AM, Chakrabarty AM (2010) Emerging Cancer Therapy: Microbial Approaches and Biotechnological Tools, 1st edn. Wiley, Singapore

Fialho AM, Salunkhe P, Manna S, Mahali S, Chakrabarty AM (2012b) Glioblastoma multiforme: novel therapeutic approaches. ISRN Neurol 2012:642345. doi: $10.5402 / 2012 / 642345$

Forbes NS (2010) Engineering the perfect (bacterial) cancer therapy. Nat Rev Cancer 10:785-94. doi: 10.1038/nrc2934

Fu G, Yin Y, Hu B, Xu G (2010) Bifidobacterium as a delivery system of functional genes for cancer gene therapy. In: Fialho AM, Chakrabarty AM (eds) Emerging Cancer Therapy: Microbial Approaches and Biotechnological tools, 1st edn. Wiley, Singapore, pp 99-118

Fu W, Chu L, Han X, Liu X RD (2008) Synergistic antitumoral effects of human telomerase reverse transcriptase-mediated dual-apoptosis-related gene vector delivered by orally attenuated Salmonella enterica Serovar Typhimurium in murine tumor models. J Gene Med 10:690-701. doi: 10.1002/jgm

Grandis V De, Bizzarri AR, Cannistraro S (2007) Docking study and free energy simulation of the complex between p53 DNA-binding domain and azurin. J Mol Recognit 20:215-226. doi: 10.1002/jmr

Hoffman RM (2010) Salmonella typhimurium mutants selected to grow only in tumors and eradicate them in nude mouse models. In: Fialho AM, Chakrabarty AM (eds) Emerging Cancer Therapy: Microbial Approaches and Biotechnological tools, 1st edn. Wiley, Singapore, pp 1-17

Huang A, Quinn H, Glover C, Henderson DC, Allen-mersh TG (2002) The presence of interleukin-2 receptor alpha in the serum of colorectal cancer patients is unlikely to result only from $T$ cell up-regulation. Cancer Immunol Immun 51:53-57. doi: $10.1007 / \mathrm{s} 00262-001-0250-6$

Jia L, Gorman GS, Coward LU, Noker PE, Mccormick D, Horn TL, Harder JB, Muzzio M (2011) Preclinical pharmacokinetics, metabolism, and toxicity of azurin-p28 ( NSC745104) a peptide inhibitor of p53 ubiquitination. Cancer Chemoth Pharm 28:513-524. doi: 10.1007/s00280-010-1518-3

Kanno S, Maeda N, Tomizawa A, Yomogida S, Katoh T, Ishikawa M (2012) The potent histone deacetylase inhibitor spiruchostatin B towards susceptible NALM-6 human B cell leukemia cells. Int J Oncol 40:1391-1396. doi: 10.3892/ijo.2011.1323

Keyhanian K, Mansoori GA, Rahimpour M (2010) Prospects for Cancer Nanotechnology 
Treatment by Azurin. Dyanmic Biochemistry, Process Biotechnology and Molecular Biology 4:48-66.

Kresowik TP, Griffith TS (2010) Bacillus Calmete-Guerin (BCG) for Urothelial Carcinoma of the Bladder. In: Fialho AM, Chakrabarty AM (eds) Emerging Cancer Therapy: Microbial Approaches and Biotechnological tools, 1st edn. Wiley, Singapore, pp 319335

Lee C, Wu C, Shiau A (2005) Systemic administration of attenuated Salmonella choleraesuis survival in the murine melanoma model. Cancer Gene Ther 12:175-184. doi: 10.1038/sj.cgt. 7700777

Lee DG, Hahm K, Park Y, Kim H, Lee W, Lim S, Seo Y, Choi C (2005). Functional and structural characteristics of anticancer peptide Pep27 analogues. 14:1-14. doi: 10.1186/1475-2867-5-21

Liu SC,Minton NP, Giaccia AJ, Brown JM (2002) Anticancer efficacy of systemically delivered anaerobic bacteria as gene therapy vectors targeting tumor hypoxia/necrosis. Gene Ther 291-296.

Lorberboum-Galski H (2011) Human toxin-based recombinant immunotoxins/chimeric proteins as a drug delivery system for targeted treatment of human diseases. Expert Opin Drug Deliv 8:605-21. doi: 10.1517/17425247.2011.566269

MacDiarmid JA, Brahmbhatt $H$ (2011) Minicells: versatile vectors for targeted drug or si/shRNA cancer therapy. Curr Opin Biotech 22:909-16. doi: 10.1016/j.copbio.2011.04.008

Mellaert L, Wei M, Anné J (2010) Live Clostridia: A powerful tool in tumor biotherapy. In: Fialho AM, Chakrabarty AM (eds) Emerging Cancer Therapy: Microbial Approaches and Biotechnological tools, 1st edn. Wiley, Singapore, pp 71-98

Micewicz, ED Jung C-L, Schaue D, Luong H, McBride WH, Ruchala P (2011) Small Azurin Derived Peptide Targets Ephrin Receptors for Radiotherapy. Int J Pept Res Ther 17:247-257. doi: 10.1007/s10989-011-9265-9

Morrissey D, O'Sullivan GC, Tangney M (2010) Tumour targeting with systemically administered bacteria. Curr Gene Ther 10:3-14.

Nemunaitis J, Cunningham C, Senzer N, Kuhn J, Litz C, Cavagnolo R, Cahill A, Clairmont C, Sznol M (2003) Expressing the Escherichia coli cytosine deaminase gene in refractory cancer patients. Cancer Gene Ther 10:737-744. doi: 10.1038/sj.cgt.7700634

Pastan I, Hassan R, FitzGerald DJ, Kreitman RJ (2007) Immunotoxin treatment of cancer. Annu Rev Med 58:221-37. doi: 10.1146/annurev.med.58.070605.115320

Patyar S, Joshi R, Byrav DSP, Prakash A, Medhi B, Das BK (2010) Bacteria in cancer 
therapy: a novel experimental strategy. J Biomed Sci 17:21. doi: 10.1186/1423-012717-21

Punj V, Bhattacharyya S, Saint-dic D, Vasu C, Cunningham EA, Graves J (2004) Bacterial cupredoxin azurin as an inducer of apoptosis and regression in human breast cancer. Oncogene 23:2367-2378.

Punj V, Gupta TK Das, Chakrabarty AM (2003) Bacterial cupredoxin azurin and its interactions with the tumor suppressor protein p53. Biochem. Biophys. Res. Comm. 312:109-114. doi: 10.1016/j.bbrc.2003.09.217

Rath CM, Janto B, Earl J, Ahmed A, Hu FZ, Hiller L, Dahlgren M, Kreft R, Yu F, Wolff JJ, Kweon HK, Christiansen MA, Kristina H, Williams RM, Ehrlich GD, Sherman DH (2011) Meta-omic characterization of the marine invertebrate microbial consortium that produces the chemotherapeutic natural product ET-743. ACS Chem Biol 743:1244-1256. doi: 10.1021/cb200244t

Rothman J, Wallecha A, Maciag P, Rivera S, Sahabi V, Paterson Y (2010) The use of Listeria monocytogenes as an active immunotherapy for the treatment of cancer. In: Fialho AM, Chakrabarty AM (eds) Emerging Cancer Therapy: Microbial Approaches and Biotechnological tools, 1st edn. Wiley, Singapore, pp 13-48

Santini S, Bizzarri AR and Salvatore C (2011) Modelling the interaction between the p53 DNA-binding domain and the p28 peptide fragment od Azurin. J Mol Recognit 24:1043-1055. Doi: 10.1002/jmr.1153

Schloissnig S, Arumugam M, Sunagawa S, Mitreva M, Tap J, Zhu A (2013) Genomic variation landscape of the human gut microbiome. Nature 493:45-50. doi: 10.1038/nature11711

Soghomonyan S a, Doubrovin M, Pike J, Luo X, Ittensohn M, Runyan JD, Balatoni J, Finn R, Tjuvajev JG, Blasberg R, Bermudes D (2005) Positron emission tomography (PET) imaging of tumor-localized Salmonella expressing HSV1-TK. Cancer Gene Ther 12:101-8. doi: 10.1038/sj.cgt.7700779

Tangney M, Pijkeren JP Van, Gahan CGM (2010) The use of Listeria monocytogenes as a DNA delivery vector for cancer gene therapy. Bioengineered 1:284-287. doi: 10.4161/bbug.1.4.11725

Taranta M, Bizzarri AR, Cannistraro S (2008) Probing the interaction between p53 and the bacterial protein azurin by single molecule force spectroscopy. J Mol Recognit 21:6370. doi: 10.1002/jmr.869

Taranta M, Bizzarri AR, Cannistraro S (2009) Modeling the interaction between the Nterminal domain of the tumor suppressor p53 and azurin. J Mol Recognit 22:215-22. doi: 10.1002/jmr.934

Tartour E, Mosseri V, Jouffroy T, Deneux L, Jaulerry C, Brunin F, Fridman WH, Rodriguez 
J (2001) Serum soluble interleukin - 2 receptor concentrations as an independent prognostic marker in head and neck cancer. Res Lett 357:1263-1264.

Taylor BN, Mehta RR, Yamada T, Lekmine F, Christov K, Chakrabarty AM, Green A, Bratescu L, Shilkaitis A, Beattie CW, Das Gupta TK (2009) Noncationic peptides obtained from azurin preferentially enter cancer cells. Cancer Res 69:537-46. doi: 10.1158/0008-5472.CAN-08-2932

Theys J, Landuyt W, Nuyts S, Mellaert L Van, Oosterom A Van, Lambin P, Anne J (2001) Specific targeting of cytosine deaminase to solid tumors by engineered Clostridium acetobutylicum. Cancer Gene Ther 8:294-297.

Walenkamp AME, Boer IGJ, Bestebroer J, Rozeveld D, Timmer-Bosscha H, Hemrika W, van Strijp JAG, de Haas CJC (2009). Staphylococcal superantigen-like 10 Inhibits CXCL12-induced. Neoplasia 11:333-344. doi: 10.1593/neo.81508

Uhua LY, Unyuan GK, Ui CH, Ongmei XY, Haoyang SC, Un TX, Aming RD (2001) Oral cytokine gene therapy against murine tumor using attenuated Salmonella thyphimurium. Int J Cancer 443:438-443. doi: 10.1002/ijc.1489

Vinodhkumar R, Song Y, Devaki T (2008) Romidepsin (depsipeptide) induced cell cycle arrest, apoptosis and histone hyperacetylation in lung carcinoma cells (A549) are associated with increase in p21 and hypophosphorylated retinoblastoma proteins expression. Biomed Pharmacother 62:85-93. doi: 10.1016/j.biopha.2007.06.002

Walenkamp AME, Bestebroer J, Boer IGJ, Kruizinga R (2010) Staphylococcal SSL5 binding to human leukemia cells inhibits cell adhesion to endothelial cells and platelets. Neoplasia 32:1-10. doi: 10.3233/CLO-2009-0486

Wang L, Chow K, Li W, Squamous E, Carcinoma C (2000) Clinical significance of serum soluble interleukin 2 receptor- $\alpha$ in esophageal squamous cell carcinoma. Clinical Cancer Res 6:1445-1451.

Weldon JE, Pastan I (2011) A guide to taming a toxin--recombinant immunotoxins constructed from Pseudomonas exotoxin $A$ for the treatment of cancer. FEBS $\mathrm{J}$ 278:4683-700. doi: 10.1111/j.1742-4658.2011.08182.x

Wolf P, Elsässer-Beile U (2010) Pseudomonas Exotoxin A-based immunotoxins for cancer. In: Chakrabarty AM, Fialho AM (eds) Emerging Cancer Therapy: Microbial Approaches and Biotechnological tools, 1st edn. Wiley, Singapore, pp 269-288

Wood LM, Vafa ZP, Paterson Y (2010) Listeria-derived ActA is an effective adjuvant for primary and metastatic tumor immunotherapy. Cancer Immunol Immunother 59:1049-1058. doi: 10.1007/s00262-010-0830-4

Xu J, Liu XS, Zhou S, Wei MQ (2009) Combination of immunotherapy with anaerobic bacteria for immunogene therapy of solid tumours. Gene Ther Mol Biol 13:36-52. 
Xu Y, Zhu L, Hu B, Fu G, Zhang H, Wang J, Xu G (2007) A new expression plasmid in Bifidobacterium longum as a delivery system of endostatin for cancer gene therapy. Cancer Gene Ther 14:151-157. doi: 10.1038/sj.cgt.7701003

Yamada T, Fialho AM, Punj V, Bratescu L, Gupta TK Das, Chakrabarty AM (2005) Internalization of bacterial redox protein azurin in mammalian cells: entry domain and specificity. Cell Microbiol 7:1418-31. doi: 10.1111/j.1462-5822.2005.00567.x

Yamada T, Goto M, Punj V, Zaborina O, Chen ML, Kimbara K, Majumdar D, Cunningham E, Das Gupta TK, Chakrabarty AM (2002) Bacterial redox protein azurin, tumor suppressor protein p53, and regression of cancer. PNAS 99:14098-103. doi: 10.1073/pnas.222539699

Yamada T, Hiraoka Y, Ikehata M, Kimbara K, Avner BS, Gupta TK Das, Chakrabarty AM (2004) Apoptosis or growth arrest: modulation of tumor suppressor p53 specificity by bacterial redox protein azurin. PNAS 101:4770-4775.

Yamada T, Christov K, Das Gupta TK BC (2011) Mechanism of action of p28, a first-inclass, non-HDM2 mediated peptide inhibitor of p53 ubiquitination. J Clin Oncol 29. p suppl; abstr e13513

Yu Y a, Zhang Q, Szalay A a (2008) Establishment and characterization of conditions required for tumor colonization by intravenously delivered bacteria. Biotechnol Bioeng 100:567-78. doi: 10.1002/bit.21785

Yuk J, Lim K, Kim K, Kim J, Lee J, Paik T, Kim J (2010) Bacillus Calmette-Guerin cell wall cytoskeleton enhances colon cancer radiosensitivity through autophagy. Autophagy $6: 46-60$.

Zaborina O, Dhiman N, Chen ML, Kostal J, Holder IA, Chakrabarty AM (2000) Secreted products of a nonmucoid Pseudomonas aeruginosa strain induce two modes of macrophage killing: external-ATP- dependent, P2Z-receptor-mediated necrosis apoptosis. Microbiology 2521-2530.

Zago C, Lefevre F, Robe P, Jarrin C, Auriol D, Vogel TM, Simonet P, Nalin R (2008) Drugs from hidden bugs: their discovery via untapped resources. Res Microbiol 159:153161. doi: 10.1016/j.resmic.2007.12.011

Zhang Y, Zhang Y, Xia L, Zhang X, Ding X, Yan F, Wu F (2012) Escherichia coli Nissle 1917 targets and restrains mouse B16 melanoma and 4T1 breast tumor through the expression of azurin protein. Appl Environ Microbiol. doi: 10.1128/AEM.01390-12 


\section{Figure Legends}

Figure 1 - Different strategies to use bacteria in cancer treatment and diagnosis. a) Engineered bacterial strain replicate inside tumor tissues and cause cancer cell death due to their own replication or due to the expression of heterologous genes, enriching the tumor microenvironment with cytotoxic products. b) Purified bacterial products can also be engineered to target specific receptors overexpressed in cancer cells or using specific products which enter preferentially in cancer cells when compared to normal tissues. c) Bacteria can also be engineered to be used for diagnosis with bioluminescence, fluorescence, magnetic resonance or positron emission.

Figure 2 - Levels of investigation of azurin-p28 roles as anticancer agents. Purified protein azurin or chemically synthesized p28 peptide derived from azurin have been studied at different levels: cytotoxicity towards multiple cancer cell lines, antiangiogenic activity, and mode of entry and action; both protein products and gene expression using bacterial vector have been investigated in animal models by using xenografted tumor models. The peptide p28 has finished phase I clinical trials supported by CDG Therapeutics Inc. (http://www.cdgti.com/; IND 77,754) in 15 stage IV cancer patients.

Figure 3 - Functional screening of metagenomic libraries to uncover new drug leads based in newly found bacterial proteins. Metagenomic libraries can be obtained from isolated and unusual environmental samples and screened for 
finding new putative anticancer drugs, targeting cancer cell growth and/or functional anti-invasive and anti-angiogenic properties. 







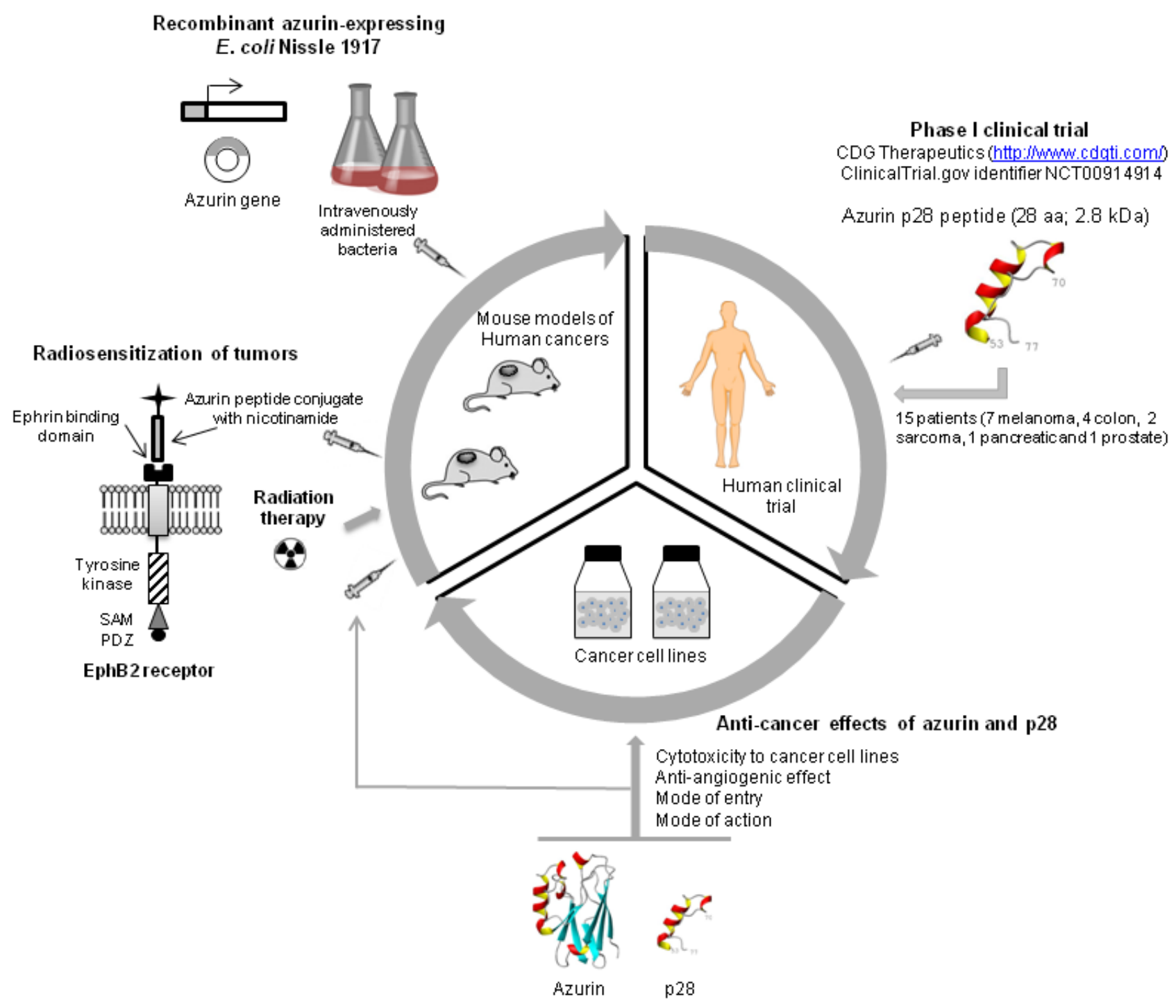

Figure 2 

